\title{
Dimension, Pseudorandomness and Extraction of Pseudorandomness*
}

\author{
Manindra Agrawal ${ }^{1}$, Diptarka Chakraborty ${ }^{1}$, Debarati Das $^{2}$, and \\ Satyadev Nandakumar ${ }^{1}$
}

1 Indian Institute of Technology Kanpur, India

\{manindra, diptarka, satyadev\}@cse.iitk.ac.in

2 Charles University in Prague, Czech Republic

debaratix710@gmail.com

\begin{abstract}
In this paper we propose a quantification of distributions on a set of strings, in terms of how close to pseudorandom a distribution is. The quantification is an adaptation of the theory of dimension of sets of infinite sequences introduced by Lutz. Adapting Hitchcock's work, we also show that the logarithmic loss incurred by a predictor on a distribution is quantitatively equivalent to the notion of dimension we define. Roughly, this captures the equivalence between pseudorandomness defined via indistinguishability and via unpredictability. Later we show some natural properties of our notion of dimension. We also do a comparative study among our proposed notion of dimension and two well known notions of computational analogue of entropy, namely HILL-type pseudo min-entropy and next-bit pseudo Shannon entropy.

Further, we apply our quantification to the following problem. If we know that the dimension of a distribution on the set of $n$-length strings is $s \in(0,1]$, can we extract out $O(s n)$ pseudorandom bits out of the distribution? We show that to construct such extractor, one need at least $\Omega(\log n)$ bits of pure randomness. However, it is still open to do the same using $O(\log n)$ random bits. We show that deterministic extraction is possible in a special case - analogous to the bitfixing sources introduced by Chor et al., which we term nonpseudorandom bit-fixing source. We adapt the techniques of Gabizon, Raz and Shaltiel to construct a deterministic pseudorandom extractor for this source.

By the end, we make a little progress towards $\mathrm{P}$ vs. BPP problem by showing that existence of optimal stretching function that stretches $O(\log n)$ input bits to produce $n$ output bits such that output distribution has dimension $s \in(0,1]$, implies $\mathrm{P}=\mathrm{BPP}$.
\end{abstract}

1998 ACM Subject Classification F.1.2 Modes of Computation

Keywords and phrases Pseudorandomness, Dimension, Martingale, Unpredictability, Pseudoentropy, Pseudorandom Extractor, Hard Function

Digital Object Identifier 10.4230/LIPIcs.FSTTCS.2015.221

\section{Introduction}

Incorporating randomness in any feasible computation is one of the basic primitives in theoretical computer science. Fortunately, any efficient (polynomial time) randomized algorithm does not require pure random bits. What it actually needs is a source that looks random to it and this is where the notion of pseudorandomness $[4,32]$ comes into picture. Since

\footnotetext{
* Research supported in part by Research-I Foundation and the European Research Council under the European Union's Seventh Framework Programme (FP/2007-2013) / ERC Grant Agreement n. 616787.

(c) (i) Manindra Agrawal, Diptarka Chakraborty, Debarati Das, and Satyadev Nandakumar; licensed under Creative Commons License CC-BY 
its introduction, pseudorandomness has been fundamental to the domain of cryptography, complexity theory and computational learning theory. Pseudorandomness is mainly a computational approach to study the nature of randomness, and computational indistinguishability [10] played a pivotal role in this. Informally, a distribution is said to be pseudorandom if no efficient algorithm can distinguish it from the uniform distribution. Another way of looking at computational indistinguishability is via the notion of unpredictability of distributions, due to Yao [32]. Informally, a distribution is unpredictable if there is no efficient algorithm that, given a prefix of a string coming from that distribution, can guess the next bit with a significant success probability. This line of research naturally posed the question of constructing algorithms that can generate pseudorandom distributions, known as pseudorandom generators. Till now we know such constructions by assuming the existence of one-way functions. It is well known that constructibility of an optimal pseudorandom generator implies complete derandomization (i.e., $\mathrm{P}=\mathrm{BPP}$ ) and exponential hardness assumption on one-way function enables us to do that. However, Nisan and Wigderson [25] showed that the existence of an exponential hard function, which is a much weaker assumption, is also sufficient for this purpose. The assumption was further weakened in [18].

In order to characterize the class of random sources, information theoretic notion of min-entropy is normally used. A computational analogue of entropy was introduced by Yao [32] and was based on compression. Håstad, Impagliazzo, Levin and Luby [12] extended the definition of min-entropy in computational settings while giving the construction of a pseudorandom generator from any one-way function. This HILL-type pseudoentropy basically extends the definition of pseudorandomness syntactically. Relations among above two types of pseudoentropy was further studied in [3]. A more relaxed notion of pseudoentropy, known as next-bit Shannon pseudoentropy, was later introduced by Haitner, Reingold and Vadhan [11] in the context of an efficient construction of a pseudorandom generator from any one-way function. In a follow up work [31], the same notion was alternatively characterized by $K L$-hardness. So far it is not clear which of the above notions is the most appropriate or whether they are at all suitable to characterize distributions in terms of the degree of pseudorandomness in it.

In this paper, we first propose an alternative measure to quantify the amount of pseudorandomness present in a distribution. This measure is motivated by the ideas of dimension [23] and logarithmic loss unpredictability [15]. Lutz used the betting functions known as gales to characterize the Hausdroff dimension of sets of infinite sequences over a finite alphabet. The definition given by Lutz cannot be carried over directly, because here we consider the distributions over finite length strings instead of sets containing infinite length strings. To overcome this difficulty, we allow "non-uniform" gales and introduce a new probabilistic notion of success of a gale over a distribution. We use this to define the dimension of a distribution. In [15], Hitchcock showed that the definition of dimension given by Lutz is equivalent to logarithmic loss unpredictability. In this paper, we show that this result can be adapted to establish a quantitative equivalence between the notion of logarithmic loss unpredictability of a distribution and our proposed notion of dimension. Roughly, this captures the essence of equivalence between pseudorandomness defined via indistinguishability and via unpredictability [32]. We show some important properties of the notion of dimension of a distribution, which eventually makes this characterization much more powerful and flexible. We also do a comparative study between our notion of dimension and two known notions of pseudoentropy, namely HILL-type pseudo min-entropy and next-bit pseudo Shannon entropy. We show that the class of distributions with high dimension is a strict superset of the class of distributions having high HILL-type pseudo min-entropy. Whereas, there is a much closer relationship between dimension and next-bit pseudo Shannon entropy. 
Once we have a quantification of pseudorandomness of a distribution, the next natural question is how to extract the pseudorandom part from a given distribution. The question is similar to the question of constructing randomness extractors which is an efficient algorithm that converts a realistic source to an almost ideal source of randomness. The term randomness extractor was first defined by Nisan and Zuckerman [26]. Unfortunately there is no such deterministic algorithm and to extract out almost all the randomness, extra $\Omega(\log n)$ pure random bits are always required $[27,28]$. There is a long line of research on construction of extractors towards achieving this bound. For a comprehensive treatment on this topic, we refer the reader to excellent surveys by Nisan and Ta-Shma [24] and Shaltiel [29]. Finally, the desired bound was achieved up to some constant factor in [20].

Coming back to the computational analogue, it is natural to study the same question in the domain of pseudorandomness. Given a distribution with dimension $s$, the problem is to output $O(s n)$ many bits that are pseudorandom. A simple argument can show that deterministic pseudorandom extraction is not possible, but it is not at all clear that how many pure random bits are necessary to serve the purpose. In this paper, we show that we need to actually involve $\Omega(\log n)$ random bits to extract out all the pseudorandomness present in a distribution. However explicit construction of one such extractor with $O(\log n)$ random bits is not known. If it is known that the given distribution has high HILL-type pseudo min-entropy, then any randomness extractor will work [3]. Instead of HILL-type pseudoentropy, even if we have Yao-type pseudo min-entropy, then also some special kind of randomness extractor (namely with a "reconstruction procedure") could serve our purpose [3]. Unfortunately both of these notions of pseudoentropy can be very small for a distribution with very high dimension. Actually the same counterexample will work for both the cases. So it is interesting to come up with an pseudorandom extractor for a class of distributions having high dimension.

As a first step towards this goal, we consider a special kind of source which we call the nonpseudorandom bit-fixing source. It is similar to the well studied notion of bit-fixing random source introduced by Chor et al. [5], for which we know the construction of a deterministic randomness extractor due to [19] and [8]. In this paper, we show that the same construction yields a deterministic pseudorandom extractor for all nonpseudorandom bit-fixing sources having polynomial-size support.

In the concluding section, we make a little progress towards the question of $\mathrm{P}$ vs. BPP by showing that in order to prove $\mathrm{P}=\mathrm{BPP}$, it is sufficient to construct an algorithm that stretches $O(\log n)$ pure random bits to $n$ bits such that the output distribution has a non-zero dimension (not necessarily pseudorandom). The idea is that using such stretching algorithm, we easily construct a hard function, which eventually gives us the most desired optimal pseudorandom generator.

Notations: In this paper, we consider the binary alphabet $\Sigma=\{0,1\}$. We denote $\operatorname{Pr}_{x \in \in_{R} D}[E]$ as $D[E]$, where $E$ is an event and $x$ is drawn randomly according to the distribution $D$. We use $U_{m}$ to denote the uniform distribution on $\Sigma^{m}$. Given a string $x \in \Sigma^{n}, x[i]$ denote the $i$-th bit of $x$ and $x[1, \ldots, i]$ denotes the first $i$ bits of $x$. Now suppose $x \in \Sigma^{n}$ and $S=\left\{s_{1}, s_{2}, \ldots, s_{k}\right\} \subseteq\{1,2, \ldots, n\}$, then by $x_{S}$, we denote the string $x\left[s_{1}\right] x\left[s_{2}\right] \ldots x\left[s_{k}\right]$.

\section{Quantification of Pseudorandomness}

In this section, we propose a quantification of pseudorandomness present in a distribution. We adapt the notion introduced by Lutz [23] of an $s$-gale to define a variant notion of success 
of an $s$-gale against a distribution $D$ on $\Sigma^{n}$. Throughout this paper, we will talk about non-uniform definitions. First, we consider the definition of pseudorandomness.

\subsection{Pseudorandomness}

We start by defining the notion of indistinguishability which we will use frequently in this paper.

- Definition 1 (Indistinguishability). A distribution $D$ over $\Sigma^{n}$ is $(S, \epsilon)$-indistinguishable from another distribution $D^{\prime}$ over $\Sigma^{n}$ (for $S \in \mathbb{N}, \epsilon>0$ ) if for every circuit $C$ of size at most $S$, $\left|D[C(x)=1]-D^{\prime}[C(x)=1]\right| \leq \epsilon$.

Now we are ready to introduce the notion of pseudorandomness.

- Definition 2 (Pseudorandomness). For a distribution $D$ over $\Sigma^{n}$ and for any $S>n,{ }^{1} \epsilon>0$,

1. (via computational indistinguishability) $D$ is said to be $(S, \epsilon)$-pseudorandom if $D$ is $(S, \epsilon)$-indistinguishable from $U_{n}$; or equivalently,

2. (via unpredictability [32]) $D$ is said to be $(S, \epsilon)$-pseudorandom if $D\left[C\left(x_{1}, \cdots, x_{i-1}\right)=\right.$ $\left.x_{i}\right] \leq \frac{1}{2}+\frac{\epsilon}{n}$ for all circuits $C$ of size at most $2 S$ and for all $i \in[n]$.

\subsection{Martingales, s-gales and predictors}

Martingales are "fair" betting games which are used extensively in probability theory (see for example, [2]). Lutz introduced a generalized notion, that of an $s$-gale, to characterize Hausdorff dimension [22] and Athreya et al. used a similar notion to characterize packing dimension[1].

- Definition 3 ([22]). Let $s \in[0, \infty)$. An $s$-gale is a function $d: \Sigma^{*} \rightarrow[0, \infty)$ such that $d(\lambda)=1$ and $d(w)=2^{-s}[d(w 0)+d(w 1)], \forall w \in \Sigma^{*}$. A martingale is a 1-gale.

The following proposition establishes a connection between $s$-gales and martingales.

- Proposition 4 ([22]). A function $d: \Sigma^{*} \rightarrow[0, \infty)$ is an s-gale if and only if the function $d^{\prime}: \Sigma^{*} \rightarrow[0, \infty)$ defined as $d^{\prime}(w)=2^{(1-s)|w|} d(w)$ is a martingale.

In order to adapt the notion of an $s$-gale to the study of pseudorandomness, we first relate it to the notion of predictors, which have been extensively used in the literature [31]. Given an initial finite segment of a string, a predictor specifies a probability distribution over $\Sigma$ for the next symbol in the string.

- Definition 5. A function $\pi: \Sigma^{*} \times \Sigma \rightarrow[0,1]$ is a predictor if for all $w \in \Sigma^{*}, \pi(w, 0)+$ $\pi(w, 1)=1$.

Note that the above definition of a predictor is not much different from the type of predictor used in Definition 2. If we have a predictor that given a prefix of a string outputs the next bit, then by invoking that predictor independently polynomially many times we can get an estimate on the probability of occurrence of 0 or 1 as the next bit and using Chernoff bound it can easily be shown that the estimation is correct up to some inverse exponential error. For the detailed equivalence, the reader may refer to [31]. In this paper,

1 Throughout this paper, we consider $S>n$ so that the circuit can at least read the full input; however reader can feel free to take any $S \in \mathbb{N}$. 
we only consider the martingales (or $s$-gales) and predictors that can be computed using non-uniform circuits and from now onwards we refer them just by martingales (or $s$-gales) and predictors. And by the size of a martingale (or an $s$-gale or a predictor), we refer the size of the circuit corresponding to that martingale (or $s$-gale or predictor).

\subsection{Conversion Between s-Gale \& Predictor}

There is an equivalence between an $s$-gale and a predictor. An early reference to this is [6]. We follow the construction given in [15].

A predictor $\pi$ induces an $s$-gale $d_{\pi}$ for each $s \in[0, \infty)$ and is defined as follows: $d_{\pi}(\lambda)=1, d_{\pi}(w a)=2^{s} d_{\pi}(w) \pi(w, a)$ for all $w \in \Sigma^{*}$ and $a \in \Sigma$; equivalently $d_{\pi}(w)=$ $2^{s|w|} \prod_{i=1}^{|w|} \pi(w[1 \cdots i-1], w[i])$ for all $w \in \Sigma^{*}$.

Conversely, an $s$-gale $d$ with $d(\lambda)=1$ induces a predictor $\pi_{d}$ defined as: if $d(w) \neq 0$, $\pi_{d}(w, a)=2^{-s} \frac{d(w a)}{d(w)}$; otherwise, $\pi_{d}(w, a)=\frac{1}{2}$, for all $w \in \Sigma^{*}$ and $a \in \Sigma$.

Hitherto, $s$-gales have been used to study the dimension of sets of infinite sequences - for an extensive bibliography, see [13] and [14]. Although in this paper, we consider distributions on finite length strings, the conversion procedure between $s$-gale and predictor will be exactly same as described above.

\subsection{Defining Dimension}

- Definition 6. An $s$-gale $d: \Sigma^{*} \rightarrow[0, \infty)$ is said to $\epsilon$-succeed over a distribution $D$ on $\Sigma^{n}$ if $D[d(w) \geq 2]>\frac{1}{2}+\epsilon$.

Note that the above definition of win of an $s$-gale is not arbitrary and reader may refer to the last portion of the proof of Theorem 13 to get some intuition behind this definition. The following lemma states the equivalence between the standard definition of pseudorandomness and the definition using martingale.

Lemma 7. There exists a constant $c^{\prime}>0$ such that for every $c>c^{\prime}$ and for any $n \in \mathbb{N}$, if a distribution $D$ over $\Sigma^{n}$ is $(S, \epsilon)$-pseudorandom then there is no martingale of size at most $(S-c)$ that $\epsilon$-succeeds on $D$. Conversely, if there is no martingale of size at most $3 S$ that $\frac{\epsilon}{n}$-succeeds on $D$, then $D$ is $(S, \epsilon)$-pseudorandom.

The proof of the above lemma follows from the fact that the martingale that wins on $D$, can act as a distinguisher circuit and conversely, if $D$ is not pseudorandom then we have a next bit predictor which can be used to construct a martingale that will win on $D$. The next definition gives a complete quantification of distributions in terms of dimension.

- Definition 8 (Dimension). The $(S, \epsilon)$-dimension of a distribution $D$ on $\Sigma^{n}$ is defined as $\operatorname{dim}_{S, \epsilon}(D)=\min \{1, \inf \{s \in[0, \infty) \mid \exists s-$ gale $d$ of size at most $S$ which $\epsilon$-succeeds on $D\}\}$. Informally, if the dimension of a distribution is $s$, we say that it is s-nonpseudorandom.

\section{Unpredictability and Dimension}

It is customary to measure the performance of a predictor utilizing a loss function [16]. The loss function determines the penalty incurred by a predictor for erring in its prediction. Let the next bit be $b$ and the probability induced by the predictor on it is $p_{b}$.

Commonly used loss functions include the absolute loss function, which penalizes the amount $1-p_{b}$; and the logarithmic loss function, which penalizes $-\log \left(p_{b}\right)$. The latter, 
which appears complicated at first glance, is intimately related to the concepts of Shannon Entropy and dimension. In this section, adapting the result of Hitchcock [15], we establish that there is an equivalence between the notion of dimension that we define in the previous section, and the logarithmic loss function defined on a predictor.

- Definition 9. The logarithmic loss function on $p \in[0,1]$ is defined to be $\operatorname{loss}(p)=-\log p$.

Using this, we define the running loss that a predictor incurs while it predicts successive bits of a string in $\Sigma^{n}$, as the sum of the losses that the predictor makes on individual bits.

- Definition 10. Let $\pi: \Sigma^{*} \times \Sigma \rightarrow[0,1]$ be a predictor.

1. The cumulative loss of $\pi$ on $w \in \Sigma^{n}$, denoted as $\operatorname{Loss}(\pi, w)$, is defined by $\operatorname{Loss}(\pi, w)=$ $\sum_{i=1}^{n} \operatorname{loss}(\pi(w[1 \ldots i-1]), w[i])$.

2. The loss rate of $\pi$ on $w \in \Sigma^{n}$ is $\operatorname{LossRate}(\pi, w)=\frac{\operatorname{Loss}(\pi, w)}{n}$.

3. The $\epsilon$-loss rate of $\pi$ over a distribution $D$ on $\Sigma^{n}$ is LossRate $_{\epsilon}(\pi, D)=\inf t+\frac{1}{n}$, where $t$ is any number in $[0,1]$ such that $D[\operatorname{LossRate}(\pi, w) \leq t]>\frac{1}{2}+\epsilon$.

Note that for a fixed $n \in \mathbb{N}$, any distribution on $\Sigma^{n}$ has loss rate between $\frac{1}{n}$ and 1 . The unpredictability of a distribution is defined as the infimum of the loss rate that any predictor has to incur on the distribution.

- Definition 11. The $(S, \epsilon)$-unpredictability of a distribution $D$ on $\Sigma^{n}$ is

$\operatorname{unpred}_{S, \epsilon}(D)=\min \left\{1, \inf \left\{\operatorname{LossRate}_{\epsilon}(\pi, D) \mid \pi\right.\right.$ is a predictor of size at most $\left.\left.S\right\}\right\}$.

With this, we can prove that dimension can equivalently be defined using unpredictability.

- Theorem 12. For any distribution $D$ on $\Sigma^{n}$, if $\operatorname{dim}_{S, \epsilon}(D) \leq s$, then $\operatorname{unpred}_{S^{2}, \epsilon}(D) \leq s$. Conversely, if $\operatorname{unpred}_{S, \epsilon}(D) \leq s$, then $\operatorname{dim}_{S^{2}, \epsilon}(D) \leq s$.

The proof of the above theorem is motivated from the proof of the equivalence between logarithmic loss unpredictability and dimension [15].

Till this point, we have given all the definitions parameterized by the circuit size $S$ and bias term $\epsilon$. However, we can naturally extend our definitions to asymptotic definitions where we consider $S$ to be any polynomial in $n$ and $\epsilon$ to be inverse of any polynomial in $n$. In that case, we will get exact equivalence between dimension and unpredictability.

\section{Properties of Dimension}

We now establish a few basic properties of our notion of dimension. We begin by exhibiting a distribution on $\Sigma^{n}$ with dimension $s$, for any $s \in(0,1]$.

First, we observe that the dimension of any distribution $D$ is the infimum of a non-empty subset of $[0,1]$ and hence the dimension of a distribution is well-defined.

Since it is clear that any distribution on $\Sigma^{n}$ has a dimension, the following theorem establishes the fact that our definition yields a nontrivial quantification of the set of distributions.

- Theorem 13. Let $s \in(0,1]$. Then for large enough $n$ and any $S>n, \epsilon>0$, there is a distribution $D$ on $\Sigma^{n}$ with $(S, \epsilon)$-dimension $s$.

Proof. Let us take a distribution $D:=U_{n}$, i.e., uniform distribution on $\Sigma^{n}$. If $s=1$, then by Lemma 7, $D$ is a distribution with the required $(S, \epsilon)$-dimension, for any $S>0$ and $\epsilon>0$.

Otherwise, assume that $s \in(0,1)$. To each string $x \in \Sigma^{n}$, we append $\left\lfloor\frac{n}{s}\right\rfloor-n$ many zeros, and denote the resulting string as $x^{\prime}$. Let $D^{\prime}\left(x^{\prime}\right)=D(x)$. For strings $y \in \Sigma \Sigma^{\left\lfloor\frac{n}{s}\right\rfloor}$ which do not terminate in a sequence of $\left\lfloor\frac{n}{s}\right\rfloor-n$ many zeros, we set $D^{\prime}(y)=0$. 
Let $\pi: \Sigma^{*} \times \Sigma \rightarrow[0,1]$ be the predictor which testifies that the $\left(S^{2}, \epsilon\right)$-unpredictability of $D \leq 1$. Define the new predictor $\pi^{\prime}: \Sigma^{*} \times \Sigma \rightarrow[0,1]$ by

$$
\pi^{\prime}(x, b)= \begin{cases}\pi(x, b) & \text { if }|x|<n, b=0,1 \\ 1 & \text { if }|x| \geq n, b=0 \\ 0 & \text { otherwise }\end{cases}
$$

For every $w \in \Sigma^{\left\lfloor\frac{n}{s}\right\rfloor}$ which is in the support of $D^{\prime} \operatorname{such}$ that $\operatorname{LossRate}(\pi, w[1 \ldots n]) \leq$ $\left(1+\epsilon_{1}-\frac{1}{n}\right)$, for any $\epsilon_{1}>0$, we have that

$$
\operatorname{LossRate}\left(\pi^{\prime}, w\right)=\frac{\operatorname{Loss}(\pi, w[1 \ldots n])}{\left\lfloor\frac{n}{s}\right\rfloor} \leq \frac{\left(1+\epsilon_{1}-\frac{1}{n}\right) n}{\left\lfloor\frac{n}{s}\right\rfloor} \leq\left(s+\epsilon^{\prime}-\frac{1}{\left\lfloor\frac{n}{s}\right\rfloor}\right),
$$

for some $\epsilon^{\prime}>0$. The last inequality holds for small enough $s / n$ and this testifies that the $\left(S^{2}, \epsilon\right)$-unpredictability (hence the $\left(S^{4}, \epsilon\right)$-dimension) of the distribution $D^{\prime}$ is at most $s$.

Now, assume that $\left(S^{4}, \epsilon\right)$-dimension of $D^{\prime}$ is less than $s$ and for some $\epsilon_{1}, 0<\epsilon_{1}<s$, there exists a $s^{\prime}$-gale $\left(s^{\prime}=s-\epsilon_{1}\right) d$ of size at most $S^{4}$ which $\epsilon$-succeeds on $D^{\prime}$. We show that this would imply that $D$ is not uniform. Now consider a string $w \in \Sigma^{\left\lfloor\frac{n}{s}\right\rfloor}$, which is in the support of $D^{\prime}$. For any $k \in n+1, \cdots,\left\lfloor\frac{n}{s}\right\rfloor, d(w[1 \ldots k]) \leq 2^{s^{\prime}} d(w[1 \ldots k-1])$ and thus $d(w) \geq 2$ will imply that $d(w[1 \ldots n]) \geq 2^{-s^{\prime}\left(\left\lfloor\frac{n}{s}\right\rfloor-n\right)+1}$. Now consider the martingale $d^{\prime}$ corresponding to the $s^{\prime}$-gale $d$. According to [22], we have $d^{\prime}\left(w^{\prime}\right)=2^{\left(1-s^{\prime}\right)\left|w^{\prime}\right|} d\left(w^{\prime}\right)$, for any string $w^{\prime} \in \Sigma^{*}$. Thus,

$$
\begin{aligned}
D^{\prime}\left[d^{\prime}(w[1 \ldots n]) \geq 2\right] & \geq D^{\prime}\left[d(w[1 \ldots n]) \geq 2^{-s^{\prime}\left(\left\lfloor\frac{n}{s}\right\rfloor-n\right)+1}\right] \\
& \geq D^{\prime}[d(w) \geq 2] \\
& >\frac{1}{2}+\epsilon .
\end{aligned}
$$

Note that $D^{\prime}\left[d^{\prime}(w[1 \ldots n]) \geq 2\right]$ is same as $D\left[d^{\prime}(x) \geq 2\right]$ or in other words $U_{n}\left[d^{\prime}(x) \geq 2\right]$, which contradicts the fact that by Markov Inequality, $U_{n}\left[d^{\prime}(x) \geq 2\right] \leq \frac{1}{2}$ and this completes the proof.

In subsequent sections, we will see how to extract pseudorandom parts from a convex combination of distributions. We will need a weaker version of the following theorem which establishes a relationship between the dimension of a convex combination of distributions in terms of the dimension of its constituent distributions.

Theorem 14. Let $D_{1}$ and $D_{2}$ be the distributions on $\Sigma^{n}$ and $\delta \in[0,1]$. Suppose $D$ is the convex combination of $D_{1}$ and $D_{2}$ defined by $D=\delta D_{1}+(1-\delta) D_{2}$. Then for any $S>n$ and $\epsilon>0, \operatorname{dim}_{S, \epsilon}(D) \geq \min \left\{\operatorname{dim}_{S, \epsilon}\left(D_{1}\right), \operatorname{dim}_{S, \epsilon}\left(D_{2}\right)\right\}$.

Proof. The claim clearly holds when $\delta$ is either 0 or 1 , so assume that $0<\delta<1$. Let $\operatorname{dim}_{S, \epsilon}\left(D_{1}\right)=s_{1}$, and $\operatorname{dim}_{S, \epsilon}\left(D_{2}\right)=s_{2}$.

For the contrary, let us assume that, $\operatorname{dim}_{S, \epsilon}(D)<\min \left\{s_{1}, s_{2}\right\}$. Now consider $s=$ $\min \left\{s_{1}, s_{2}\right\}-\epsilon_{1}$, for some $\epsilon_{1}, 0<\epsilon_{1}<\min \left\{s_{1}, s_{2}\right\}$. Then there exists an $s$-gale $d$ of size at most $S$ such that $D[d(w) \geq 2]>\frac{1}{2}+\epsilon$.

Let the string $w$ for which $d(w) \geq 2$ holds be $w_{i}, 1 \leq i \leq k$ and the corresponding probabilities in $D$ be $p\left(w_{i}\right), 1 \leq i \leq k$. Let $q\left(w_{i}\right)$ and $r\left(w_{i}\right), 1 \leq i \leq k$, be the corresponding probabilities in $D_{1}$ and $D_{2}$ respectively. So, $\sum_{i=1}^{k} p\left(w_{i}\right)>\frac{1}{2}+\epsilon$, where $p\left(w_{i}\right)=\delta q\left(w_{i}\right)+(1-$ $\delta) r\left(w_{i}\right), 1 \leq i \leq k$. Now, since $\operatorname{dim}_{S, \epsilon}\left(D_{2}\right)=s_{2}$, we have that $r\left(w_{1}\right)+\cdots+r\left(w_{k}\right) \leq \frac{1}{2}+\epsilon$. Thus $q\left(w_{1}\right)+\cdots+q\left(w_{k}\right)>\frac{1}{2}+\epsilon$ implying $\operatorname{dim}_{S, \epsilon}\left(D_{1}\right)<s_{1}$, which is a contradiction. 
If we just concentrate on pseudorandom distributions, then by replacing $s$-gales with martingales in the proof of the above theorem, we will get the following lemma, which will be used in Section 6.1.

- Lemma 15. Let $D_{1}$ and $D_{2}$ be the $(S, \epsilon)$-pseudorandom distributions on $\Sigma^{n}$ for any $S>n, \epsilon>0$ and $\delta \in[0,1]$. Suppose there exists a distribution $D$ which can be expressed as $D=\delta D_{1}+(1-\delta) D_{2}$, then $D$ is also $(S, \epsilon)$-pseudorandom

However, it is easy to see that convex combinations of distributions may have larger dimension than any of its constituents. For example, let us consider a $n \in \mathbb{N}$ and take the distribution $U_{n}$. Now take two distributions on $\Sigma^{n+1}$, namely, $D_{1}$ produced by the 0-dilution (padding each string with a 0 at the end) of $U_{n}$ and $D_{2}$ produced by the 1-dilution (padding each string with a 1 at the end) of $U_{n}$. Then $D=0.5 D_{1}+0.5 D_{2}$ is nothing but $U_{n+1}$ and has dimension which exceeds the dimensions of $D_{1}$ and $D_{2}$ by $\frac{1}{n}$.

- Theorem 16. Let $D, D_{1}$ and $D_{2}$ be the distributions on $\Sigma^{n}$, and consider $S>n, \epsilon>0$ and $\delta \in[0,1]$. Suppose further that $\operatorname{dim}_{S, \epsilon}\left(D_{1}\right)=s_{1}$. Now if $D=(1-\delta) D_{1}+\delta D_{2}$, then $\operatorname{dim}_{S,(\epsilon+\delta)}(D) \geq s_{1} .^{2}$

The proof of the above theorem is similar to that of Theorem 14. If we follow the proof of Theorem 16 with martingale instead of $s$-gale, we get the following weaker version of the above theorem, which we will require in the construction of deterministic extractor for a special kind of sources in Section 6.1.

- Lemma 17. Let $D, D_{1}$ and $D_{2}$ be the distributions on $\Sigma^{n}$, and consider $S>n, \epsilon>0$ and $\delta \in[0,1]$. If $D_{1}$ is $(S, \epsilon)$-pseudorandom and $D=(1-\delta) D_{1}+\delta D_{2}$, then $D$ is $(S, \epsilon+\delta)$ pseudorandom as well.

The following theorem shows that in order for a distribution to have dimension less than 1 , it is not sufficient to have a few positions where we can successfully predict - it is necessary that these positions occur often.

- Theorem 18. For large enough $n$ and for any $S>n$ and $\epsilon>0$, there is a distribution $D_{n}$ on $\Sigma^{n}$ such that $\operatorname{dim}_{S, \epsilon}\left(D_{n}\right)=1$, but is not $(S, \epsilon)$-pseudorandom.

\section{Pseudoentropy and Dimension}

In this section we study the relation between our notion of dimension and different variants of computational or pseudo (min/Shannon) entropy.

\subsection{High HILL-type pseudo min-entropy implies high dimension}

For a distribution $D$, min-entropy of $D$ is defined as $H_{\infty}(D)=\min _{w}\{\log (1 / D[w])\}$. We start with the standard definition of computational min-entropy, as given by [12].

- Definition 19 (HILL-type pseudo min-entropy). A distribution $D$ on $\Sigma^{n}$ has $(S, \epsilon)$-HILL-type pseudo min-entropy (or simply $(S, \epsilon)$-pseudo min-entropy) at least $k$, denoted as $H_{\infty}^{H I L L, S, \epsilon} \geq$ $k$ if the there exists a distribution $D^{\prime}$ such that

1. $H_{\infty}\left(D^{\prime}\right) \geq k$, and

2. $D^{\prime}$ is $(S, \epsilon)$-indistinguishable from the distribution $D$.

2 Note that bias term in the dimension of $D_{1}$ depends on $\delta$. 
Several other definitions of pseudo min-entropy (metric-type, Yao-type or compression type) are there in the literature. We refer the reader to [3] for a comprehensive treatment on different definitions and the connections between them. In the remaining potion of this subsection, we focus only on HILL-type pseudo min-entropy. Now we state the main result of this subsection.

- Theorem 20. There exists a constant $c^{\prime}>0$ such that for any $c>c^{\prime}$, for every distribution $D$ on $\Sigma^{n}$ and for any $S>n, \epsilon>0$, if $H_{\infty}^{H I L L,(S+c), \epsilon}(D) \geq$ sn, then $\operatorname{dim}_{S, \epsilon}(D) \geq s$

Proof. The theorem is a consequence of the following claim.

- Claim 21. For every distribution $X$ on $\Sigma^{n}$, if $H_{\infty}(X)=k$ then $\operatorname{dim}_{S, \epsilon}(X) \geq k / n$, for any values of $S$ and $\epsilon>0$.

Now observe that if a distribution $D$ is $(S+c, \epsilon)$-indistinguishable from another distribution $D^{\prime}$, then $\operatorname{dim}_{S, \epsilon}(D)=\operatorname{dim}_{S, \epsilon}\left(D^{\prime}\right)$ as otherwise the $s$-gale which $\epsilon$-succeeds over exactly one of them, acts as a distinguishing circuit. This fact along with Claim 21 completes the proof.

It only remains to establish Claim 21.

Proof of Claim 21. Let us first take $s=k / n$. Now for the sake of contradiction, let us assume that there exists an $s$-gale $d$ that $\epsilon$-succeeds over $X$, i.e., $X[d(w) \geq 2]>\frac{1}{2}+\epsilon$. Now consider the set $S:=\{w \mid d(w) \geq 2\}$. As $H_{\infty}(X)=k,|S|>2^{s n-1}+2^{s n} . \epsilon$. By taking the corresponding martingale $d^{\prime}$ according to the Proposition 4, we have that for any $w \in S$, $d^{\prime}(w) \geq 2^{(1-s) n+1}$ and as a consequence, $U_{n}\left[d^{\prime}(w) \geq 2^{(1-s) n+1}\right]>2^{s n-n-1}+2^{s n-n} . \epsilon$, which contradicts the fact that by Markov inequality, $U_{n}\left[d^{\prime}(w) \geq 2^{(1-s) n+1}\right] \leq 2^{s n-n-1}$.

The converse direction of the statement of Theorem 20 is also true if the distribution under consideration is pseudorandom. If the converse is true then we can apply any randomness extractor to get pseudorandom distribution from any distribution having high dimension [3]. However, we should always be careful about the circuit size with respect to which we call the output distribution pseudorandom. Unfortunately, in general the converse is not true.

Counterexample for the converse: Suppose one-way functions exist, then it is well-known that we can construct a pseudorandom generator $G: \Sigma^{l} \rightarrow \Sigma^{m}$ such that $m$ is any polynomial in $l$, say $m=l^{3}$. For the definitions of one-way function, pseudorandom generator and the construction of pseudorandom generator with polynomial stretch from any one-way function, interested reader may refer to $[9,12,31]$. Now consider the distribution $D:=\left(G\left(U_{l}\right), U_{l}\right)$. For large enough $l$, using the argument similar to the proof of Theorem 13, it can easily be shown that the distribution $D$ has dimension almost 1 as the distribution on the first $m$ bits are pseudorandom, but pseudo min-entropy is not larger than $l$.

\subsection{Equivalence between dimension and next-bit pseudo Shannon entropy}

We will use standard notions and notations of information theory (e.g., Shannon entropy, KL divergence) without defining them. Readers may refer to a book by Cover and Thomas [7] for the definitions.

In the last subsection, we have talked about pseudo min-entropy. In similar fashion, one can also define pseudo Shannon entropy and a natural generalization of it is conditional pseudo Shannon entropy $[17,11,31]$. 
- Definition 22 (Conditional pseudo Shannon entropy). Suppose $Y$ is a random variable jointly distributed with $X . Y$ is said to have $(S, \epsilon)$-conditional pseudo Shannon entropy at least $k$ given $X$ if there exists a distribution $Z$ jointly distributed with $X$ such that

1. $H(Z \mid X) \geq k$, and

2. $(X, Y)$ and $(X, Z)$ are $(S, \epsilon)$-indistinguishable.

The following is the variant of pseudoentropy that we are looking for in this subsection and was introduced by Haitner et al. [11].

- Definition 23 (Next-bit pseudo Shannon entropy). A random variable $X=\left(X_{1}, X_{2}, \cdots, X_{n}\right)$ taking values in $\Sigma^{n}$ has $(S, \epsilon)$-next-bit pseudo Shannon entropy at least $k$, denoted as $H^{\text {next }, S, \epsilon}(X) \geq k$ if there exist random variables $\left(Y_{1}, Y_{2}, \cdots, Y_{n}\right)$ such that

1. $\sum_{i} H\left(Y_{i} \mid X_{1}, \cdots, X_{i-1}\right) \geq k$, and

2. for all $1 \leq i \leq n,\left(X_{1}, \cdots, X_{i-1}, X_{i}\right)$ and $\left(X_{1}, \cdots, X_{i-1}, Y_{i}\right)$ are $(S, \epsilon)$-indistinguishable. Later, Vadhan and Zheng [31] provided an alternative characterization of conditional pseudo Shannon entropy by showing an equivalence between it and KL-hardness (defined below). We use this alternative characterization extensively for our purpose.

- Definition 24 (KL-hardness). Suppose $(X, Y)$ is a $\Sigma^{n} \times \Sigma$-valued random variable and $\pi$ be any predictor. Then $\pi$ is said to be a $\delta$-KL-predictor of $Y$ given $X$ if $\mathbf{K L}\left(X, Y \| X, C_{\pi}\right) \leq \delta$ where $C_{\pi}(y \mid x)=\pi(x, y)$ for all $x \in \Sigma^{n}$ and $y \in \Sigma$.

Moreover, $Y$ is said to be $(S, \delta)$-KL-hard given $X$ if there is no predictor $\pi$ of size at most $S$ that is a $\delta$-KL-predictor of $Y$ given $X$.

The following theorem provides the equivalence among KL-hardness and conditional pseudo Shannon entropy of a distribution.

- Theorem 25 ([31]). For a $\Sigma^{n} \times \Sigma$-valued random variable $(X, Y)$ and for any $\delta>0$, $\epsilon>0$,

1. If $Y$ is $(S, \delta)$-KL-hard given $X$, then for every $\epsilon>0, Y$ has $\left(S^{\prime}, \epsilon\right)$-conditional pseudo Shannon entropy at least $H(Y \mid X)+\delta-\epsilon$, where $S^{\prime}=S^{\Omega(1)} /$ poly $(n, 1 / \epsilon)$.

2. Conversely, if $Y$ has $(S, \epsilon)$-conditional pseudo Shannon entropy at least $H(Y \mid X)+\delta$, then for every $\sigma>0, Y$ is $\left(S^{\prime}, \delta^{\prime}\right)$-KL-hard given $X$, where $S^{\prime}=\min \left\{S^{\Omega(1)} /\right.$ poly $\log (1 / \sigma)$, $\Omega(\sigma / \epsilon)\}$ and $\delta^{\prime}=\delta-\sigma$.

Now we are ready to state the main theorem of this subsection which conveys the fact that the distributions with high dimensions also have high next-bit pseudo Shannon entropy.

- Theorem 26. For any $\epsilon^{\prime}>0$, there exists a $n^{\prime} \in \mathbb{N}$ such that for any $n \geq n^{\prime}$ and $S>n$, $\epsilon>0$, for every distribution $D$ on $\Sigma^{n}$, if $\operatorname{dim}_{S, \epsilon}(D)>\frac{2 s}{1-2 \epsilon}+\epsilon^{\prime}$, then $H^{\text {next, }, S^{\prime}, \epsilon}(D)>s n$, where $S^{\prime}=S^{\Omega(1)} / \operatorname{poly}(n)$.

To prove the above theorem, we first break $D$ with dimension greater than $\frac{2 s}{1-2 \epsilon}+\epsilon^{\prime}$ into 1-bit blocks, i.e., $D=\left(D_{1}, D_{2}, \cdots, D_{n}\right)$ and then by applying Item 1 of Theorem 25 , we argue that next-bit pseudoentropy is at most $s n$ implies unpredictability is at most $\frac{2 s}{1-2 \epsilon}+\epsilon^{\prime}$ and thus get a contradiction.

On the contrary, for the other direction, the following weaker version can easily be proven.

- Theorem 27. For any $\epsilon^{\prime}>0$, there exists a $n^{\prime} \in \mathbb{N}$ such that for any $n \geq n^{\prime}$ and $S>n$, $\epsilon>0$, for every distribution $D$ on $\Sigma^{n}$, if $H^{\text {next }, S, \epsilon}(D)>s n$, then $\operatorname{dim}_{S^{\prime}, \epsilon}(D)>s-\frac{1}{2}-\epsilon^{\prime \prime}$, where $S^{\prime}=\min \left\{S^{\Omega(1)} /\right.$ poly $\left.\log \left(1 / \epsilon^{\prime}\right), \Omega\left(\epsilon^{\prime} / \sqrt{\epsilon}\right)\right\}$ and $\epsilon^{\prime \prime}=\epsilon^{\prime}-\epsilon$.

Technique used in the proof of the above theorem has a similar essence as of Theorem 26. The above two theorems can easily be extended to the asymptotic world in a natural way. 


\section{Pseudorandom Extractors \& Lower Bound}

We now introduce the notion of pseudorandom extractor similar to the notion of randomness extractor. Intuitively, a randomness extractor is a function that outputs almost random (statistically close to uniform) bits from weakly random sources, which need not be close to the uniformly random source. Two distributions $X$ and $Y$ on a set $\Lambda$ are said to be $\epsilon-$ close (statistically close) if $\max _{S \subseteq \Lambda}\{|\operatorname{Pr}[X \in S]-\operatorname{Pr}[Y \in S]|\} \leq \epsilon$ or equivalently $\frac{1}{2} \sum_{x \in \Lambda}|\operatorname{Pr}[X=x]-\operatorname{Pr}[Y=x]| \leq \epsilon$.

- Definition 28 (Deterministic Randomness Extractor). A function $E: \Sigma^{n} \rightarrow \Sigma^{m}$ is said to be a deterministic $\epsilon$-extractor for a class of distributions $\mathcal{C}$ if for every distribution $X$ on $n$-bit strings in $\mathcal{C}$, the distribution $E(X)$ is $\epsilon$-close to $U_{m}$.

Likewise, a seeded $\epsilon$-extractor is defined and the only difference is that now it takes a $d$-bit string chosen according to an uniform distribution, as an extra input. Before going further, we mention that for ease of presentation, now onwards we will only talk about asymptotic versions of the definitions and results derived so far related to pseudorandomness and dimension. We now define the notion of a pseudorandom extractor, the purpose of which is to extract out pseudorandom distribution from a given distribution.

- Definition 29 (Pseudorandom Extractor). A function $E: \Sigma^{n} \rightarrow \Sigma^{m}$ is said to be a deterministic pseudorandom extractor for a class of distributions $\mathcal{C}$ if for every distribution $X$ on $n$-bit strings in $\mathcal{C}, E(X)$ is pseudorandom.

A function $E: \Sigma^{n} \times \Sigma^{d} \rightarrow \Sigma^{m}$ is said to be a seeded pseudorandom extractor for a class of distributions $\mathcal{C}$ if for every distribution $X$ on $n$-bit strings in $\mathcal{C}, E\left(X, U_{d}\right)$ is pseudorandom.

In this section, we will concentrate on the class of distributions having dimension at least $s$. It is clear from the results stated in Section 5.1 that this class of distribution is a strict superset of the class of distributions with HILL-type pseudo min-entropy at least sn, for which any randomness extractor will act as a pseudorandom extractor [3]. Thus it is natural to ask the following.

- Question 1. For any $s \in(0,1]$, does there exist a deterministic/seeded pseudorandom extractor for the class of distributions on $\Sigma^{n}$ having dimension at least $s$ ?

Just like the the case of randomness extraction, one can easily argue that deterministic pseudorandom extraction is not possible ${ }^{3}$. Now the most common question comes next is that what the lower bound on the seed length will be. We answer to this question in the following theorem.

- Theorem 30. Suppose for any $s \in(0,1], E: \Sigma^{n} \times \Sigma^{d} \rightarrow \Sigma^{m}$ be a seeded pseudorandom extractor for the class of distributions on $\Sigma^{n}$ having dimension at least $s$ and for some $\delta>0$, $m=(s n)^{\delta}$. Then $d=\Omega(\log n)$.

Proof. For the sake of contradiction, let us assume that $d=o(\log n)$. Now by doing a walk according to the output distribution on an odd-length cycle, we achieve the following claim.

- Claim 31. There is a deterministic $\frac{1}{\sqrt[4]{m}}$-extractor $E^{\prime}: \Sigma^{m} \rightarrow \Sigma^{\frac{\log m}{4}}$ for all pseudorandom distributions on $\Sigma^{m}$.

3 Suppose $E: \Sigma^{n} \rightarrow \Sigma$ is a deterministic pseudorandom extractor, then there exists $x \in \Sigma$ such that $\left|E^{-1}(x)\right| \geq 2^{n-1}$. Thus $E$ is not a pseudorandom extractor for a source $D$ that is a uniform distribution on $E^{-1}(x)$ and by Claim $21, \operatorname{dim}(D) \geq(1-1 / n)$. 
Now construct the following function Ext : $\Sigma^{n} \times \Sigma^{d} \rightarrow \Sigma^{c \log n}$ for some constant $c>0$ such that $\operatorname{Ext}(x, y)=E^{\prime}(E(x, y))$ for all $x \in \Sigma^{n}, y \in \Sigma^{d}$. The function $E x t$ is a seeded $\frac{1}{(s n)^{\delta / 4}}$-extractor with $d=o(\log n)$, but it is well known due to [28](Theorem 1.9) that any such randomness extractor must satisfy $d=\Omega(\log n)$ and hence we get a contradiction.

However, the question on constructing an explicit or polynomial time computable seeded pseudorandom extractor with seed length $O(\log n)$ is still open and next, we formally pose this question.

- Question 2. For any $s \in(0,1]$, can one construct a seeded pseudorandom extractor $E: \Sigma^{n} \times \Sigma^{d} \rightarrow \Sigma^{m}$ in polynomial time, for the class of distributions on $\Sigma^{n}$ having dimension at least $s$ such that $m=(\text { sn })^{\delta}$ for some $\delta>0$ and $d=O(\log n)$ ?

In the next part of this section, we will see a special type of nonpseudorandom source and give an explicit construction of deterministic pseudorandom extractor for that particular type of source. Before proceeding further, we want to mention that it is also very interesting to consider nonpseudorandom distributions samplable by poly-size circuits, which is a natural extension of another special type of source called samplable source studied in [30]. By following the argument in [30], we can observe that the existence of deterministic pseudorandom extractor implies separation between deterministic complexity classes and non-uniform circuit classes which is not known so far. Nevertheless, it is still natural to ask the question of constructing explicit extractor using $O(\log n)$ amount of extra randomness for this special kind of source. We do not know any such result so far, but in Section 7 we will see that if some distribution is samplable using very few $(O(\log n))$ random bits, then it is possible to extract out all the pseudorandom bits using extra $O(\log n)$ random bits.

\subsection{Deterministic Pseudorandom Extractor for Nonpseudorandom Bit-fixing Sources}

In Section 4 while proving Theorem 13, we have introduced a special type of nonpseudorandom distribution which looks similar to the $(n, k)$-bit-fixing source defined as a distribution $X$ over $\Sigma^{n}$ such that there exists a subset $I=\left\{i_{1}, \ldots, i_{k}\right\} \subseteq\{1, \ldots, n\}$ where all the bits at the indices of $I$ are independent and uniformly chosen and rest of the bits are completely fixed. This distribution was introduced by Chor et al.[5]. Now we define an analogous notion for the class of nonpseudorandom distributions, which we term nonpseudorandom bit-fixing sources.

- Definition 32 (Nonpseudorandom Bit-fixing Source). Let $s \in(0,1)$. For sufficiently large $n$ and $\epsilon>0$, a distribution $D_{n}$ over $\Sigma^{n}$ with dimension $s$ is an $(n, s, \epsilon)$-nonpseudorandom bit-fixing source if there exists a subset $I=\left\{i_{1}, \ldots, i_{\lceil s n\rceil}\right\} \subseteq\{1, \ldots, n\}$ such that all the bits at the indices of $I$ come from an $\epsilon$-pseudorandom distribution and rest of the bits are fixed.

We devote the rest of the section to achieve an affirmative answer to the question of constructing deterministic pseudorandom extractor for the nonpseudorandom bit-fixing sources. For this purpose, we show that a careful analysis of the technique used in the construction of the deterministic randomness extractor for bit-fixing random sources by Gabizon, Raz and Shaltiel [8] will lead us to the desired deterministic pseudorandom extractor.

- Theorem 33. There exists a constant $c>0$ such that for any $s \in(0,1]$ and for large enough $n, 0<\epsilon<\frac{1}{\sqrt{n}}$, there is an explicit deterministic pseudorandom extractor $E: \Sigma^{n} \rightarrow \Sigma^{m}$ for all $(n, s, \epsilon)$-nonpseudorandom bit-fixing sources having polynomial-size support, where $m=(s n)^{\Omega(1)}$. 
We first extract $O(\log s n)$ amount of almost random bits and then use the same as seed in the seeded extractor. To use the seeded extractor, we modify the source such that it becomes independent of the random bits extracted.

\section{$7 \quad$ Approaching Towards $\mathrm{P}=\mathrm{BPP}$}

We now show that if there is an exponential time computable algorithm $G: \Sigma^{O(\log n)} \rightarrow \Sigma^{n}$ where the output distribution has dimension $s(s>0)$, then this will imply $\mathrm{P}=\mathrm{BPP}$. We refer to this algorithm $G$ as optimal nonpseudorandom generator. To prove the main result of this section, we use the following theorem proved by Impagliazzo and Wigderson.

- Theorem 34 ([18]). Suppose there is a language $L$ in $\operatorname{EXP}$ and $\exists \delta>0$ such that $L$ on inputs of length $n$ cannot be solved by circuits of size at most $2^{\delta n}$. Then there exists a language $L^{\prime}$ in EXP and $\exists \delta^{\prime}>0$ such that $L^{\prime}$ on inputs of length $n$ is $\left(2^{\delta^{\prime} n}, 1 / 2^{\delta^{\prime} n}\right)$-hard and as a consequence optimal pseudorandom generator exists.

Now we use the above theorem in the proof of the following result.

- Theorem 35. Consider any $s \in(0,1]$ and $c>0$. If there exists an algorithm $G_{n}$ : $\Sigma^{c \log n} \rightarrow \Sigma^{n}$ computable in $2^{O(\log n)}$ such that for sufficiently large $n, \operatorname{dim}\left(G_{n}\left(U_{c} \log n\right)\right) \geq s$, then $\mathrm{P}=\mathrm{BPP}$.

Proof. Suppose $X:=G_{n}\left(U_{c \log n}\right)$. If $\operatorname{dim}(X)=s>0$, then there must be a subset of indices $S \subseteq\{1,2, \cdots, n\}$ such that $|S|=\log n$ and for any $i \in S$, loss incurred by any polynomial size predictor at $i$-th bit position is non-zero or in other words, for any poly-size circuit $C, X\left[C\left(x_{1}, \cdots, x_{i-1}\right)=x_{i}\right]<1$. Otherwise according to Theorem 12 and by the argument used in the proof of Theorem 18, one can show that $\operatorname{dim}(X)=0$, for large enough $n$. Suppose $S$ contains first $\log n$ many such indices. Also assume that $S=\left\{i_{1}, i_{2}, \cdots, i_{\log n}\right\}$ and $i_{1}<i_{2}<\cdots<i_{\log n}$. Now we define two languages $L_{0}$ and $L_{1}$ as follows: for $j=0,1$, $L_{j}:=\left\{y \in \Sigma^{\log n-1} \mid \exists x \in \Sigma^{n}\right.$ in the support of $G_{n}$ and $\left.x_{S}=j y\right\}$.

First of all, note that as $i_{1} \in S$, none of $L_{0}$ and $L_{1}$ is a constant function. Now clearly either $L_{0}$ or $L_{1}$ is the language that satisfies all the conditions of Theorem 34 [18]. Otherwise, there exists a predictor circuit of size at most $2^{\delta \log n}$, for some $\delta>0$, i.e., polynomial in $n$, by which we can predict $i_{\log n}$-th bit position or loss incurred by that predictor at $i_{\log n}$ th bit position will be zero implying $i_{\log n} \notin S$ which is a contradiction. Thus either $L_{0}$ or $L_{1}$ can be used to construct an optimal pseudorandom generator and which eventually implies $\mathrm{P}=\mathrm{BPP}$.

Acknowledgements. The authors thank Somenath Biswas for helpful discussions and comments. The second author also thanks Andrej Bogdanov for suggesting the study of the notion of pseudoentropy.

\section{References}

1 K. B. Athreya, J. M. Hitchcock, J. H. Lutz, and E. Mayordomo. Effective strong dimension, algorithmic information, and computational complexity. SIAM Journal on Computing, 37:671-705, 2007.

2 K. B. Athreya and S. N. Lahiri. Measure Theory and Probability Theory. Springer Verlag, 2006 . 
3 Boaz Barak, Ronen Shaltiel, and Avi Wigderson. Computational analogues of entropy. In Sanjeev Arora, Klaus Jansen, José D. P. Rolim, and Amit Sahai, editors, RANDOMAPPROX, volume 2764 of Lecture Notes in Computer Science, pages 200-215. Springer, 2003.

4 Manuel Blum and Silvio Micali. How to generate cryptographically strong sequences of pseudo-random bits. SIAM J. Comput., 13(4):850-864, November 1984.

5 Benny Chor, Oded Goldreich, Johan Håstad, Joel Freidmann, Steven Rudich, and Roman Smolensky. The bit extraction problem or t-resilient functions, 1985.

6 T. Cover. Universal gambling schemes and the complexity measures of Kolmogorov and Chaitin. Technical Report 12, Stanford University Department of Statistics, October 1974.

7 Thomas M. Cover and Joy A. Thomas. Elements of Information Theory (Wiley Series in Telecommunications and Signal Processing). Wiley-Interscience, 2006.

8 Ariel Gabizon, Ran Raz, and Ronen Shaltiel. Deterministic extractors for bit-fixing sources by obtaining an independent seed, 2005.

9 Oded Goldreich. The Foundations of Cryptography - Volume 1, Basic Techniques. Cambridge University Press, 2001.

10 Shafi Goldwasser and Silvio Micali. Probabilistic encryption. J. Comput. Syst. Sci., 28(2):270-299, 1984.

11 Iftach Haitner, Omer Reingold, and Salil P. Vadhan. Efficiency improvements in constructing pseudorandom generators from one-way functions. In Proceedings of the 42nd ACM Symposium on Theory of Computing, STOC 2010, Cambridge, Massachusetts, USA, 5-8 June 2010, pages 437-446, 2010.

12 Johan Håstad, Russell Impagliazzo, Leonid A. Levin, and Michael Luby. A pseudorandom generator from any one-way function. SIAM Journal on Computing, 28(4):1364-1396, 1999.

13 J. M. Hitchcock. Effective Fractal Dimension Bibliography, http://www.cs.uwyo.edu/ $\sim$ jhitchco/bib/dim.shtml (current April, 2011).

14 J. M. Hitchcock. Resource Bounded Measure - Bibliography, http://www.cs.uwyo.edu/ $\sim$ jhitchco/bib/rbm.shtml (current April, 2011).

15 J. M. Hitchcock. Fractal dimension and logarithmic loss unpredictability. Theoretical Computer Science, 304(1-3):431-441, 2003.

16 John M. Hitchcock. Fractal dimension and logarithmic loss unpredictability, 2004.

17 Chun-Yuan Hsiao, Chi-Jen Lu, and Leonid Reyzin. Conditional computational entropy, or toward separating pseudoentropy from compressibility. In Moni Naor, editor, Advances in Cryptology - EUROCRYPT 2007, 26th Annual International Conference on the Theory and Applications of Cryptographic Techniques, Barcelona, Spain, May 20-24, 200\%, Proceedings, volume 4515 of Lecture Notes in Computer Science, pages 169-186. Springer, 2007.

18 Russell Impagliazzo and Avi Wigderson. $\mathrm{P}=\mathrm{BPP}$ unless $\mathrm{E}$ has sub-exponential circuits: Derandomizing the xor lemma (preliminary version). In In Proceedings of the 29th STOC, pages 220-229. ACM Press, 1996.

19 Jesse Kamp and David Zuckerman. Deterministic extractors for bit-fixing sources and exposure-resilient cryptography. In In Proceedings of the 44th Annual IEEE Symposium on Foundations of Computer Science, pages 92-101, 2003.

20 Chi-Jen Lu, Omer Reingold, Salil P. Vadhan, and Avi Wigderson. Extractors: optimal up to constant factors. In Lawrence L. Larmore and Michel X. Goemans, editors, STOC, pages 602-611. ACM, 2003.

21 J. H. Lutz. Gales and the constructive dimension of individual sequences. In Proceedings of the 27th International Colloquium on Automata, Languages, and Programming, pages 902-913, 2000. Revised as [22].

22 J. H. Lutz. The dimensions of individual strings and sequences. Information and Computation, 187:49-79, 2003. Preliminary version appeared as [21]. 
23 Jack H. Lutz. Dimension in complexity classes. SIAM J. Comput., 32(5):1236-1259, 2003.

24 Noam Nisan and Amnon Ta-Shma. Extracting randomness: A survey and new constructions. J. Comput. Syst. Sci., 58(1):148-173, 1999.

25 Noam Nisan and Avi Wigderson. Hardness vs randomness. J. Comput. Syst. Sci., 49(2):149 167, 1994.

26 Noam Nisan and David Zuckerman. More deterministic simulation in logspace. In S. Rao Kosaraju, David S. Johnson, and Alok Aggarwal, editors, Proceedings of the Twenty-Fifth Annual ACM Symposium on Theory of Computing, May 16-18, 1993, San Diego, CA, USA, pages 235-244. ACM, 1993.

27 Noam Nisan and David Zuckerman. Randomness is linear in space. Journal of Computer and System Sciences, 52:43-52, 1996.

28 Jaikumar Radhakrishnan and Amnon Ta-Shma. Bounds for dispersers, extractors, and depth-two superconcentrators. SIAM Journal on Discrete Mathematics, 13:2000, 2000.

29 Ronen Shaltiel. Recent developments in explicit constructions of extractors. Bulletin of the EATCS, 77:67-95, 2002.

30 Luca Trevisan and Salil P. Vadhan. Extracting randomness from samplable distributions. In FOCS, pages 32-42. IEEE Computer Society, 2000.

31 Salil P. Vadhan and Colin Jia Zheng. Characterizing pseudoentropy and simplifying pseudorandom generator constructions. In Proceedings of the 44th Symposium on Theory of Computing Conference, STOC 2012, New York, NY, USA, May 19-22, 2012, pages 817836, 2012.

32 Andrew C. Yao. Theory and application of trapdoor functions. In Proceedings of the 23rd Annual Symposium on Foundations of Computer Science, SFCS'82, pages 80-91, Washington, DC, USA, 1982. IEEE Computer Society. 